\title{
Liquid-liquid phase transition for an attractive isotropic potential with wide repulsive range
}

\author{
Gianpietro Malescio, ${ }^{1}$ Giancarlo Franzese, ${ }^{2,3}$ Anna Skibinsky, ${ }^{3}$ Sergey V. Buldyrev, ${ }^{3,4}$ and H. Eugene Stanley ${ }^{3}$ \\ ${ }^{1}$ Dipartimento di Fisica, Università di Messina and Istituto Nazionale Fisica della Materia, 98166 Messina, Italy \\ ${ }^{2}$ Departament de Física Fonamental, Facultat de Física, Universitat de Barcelona, Diagonal 647, 08028 Barcelona, Spain \\ ${ }^{3}$ Center for Polymer Studies and Department of Physics, Boston University, Boston, Massachusetts 02215, USA \\ ${ }^{4}$ Department of Physics, Yeshiva University, $500 \mathrm{~W}$ 185th St., New York, New York 10040,USA
}

(Received 10 November 2004; revised manuscript received 8 February 2005; published 17 June 2005)

\begin{abstract}
We investigate how the phase diagram of a repulsive soft-core attractive potential, with a liquid-liquid phase transition in addition to the standard gas-liquid phase transition, changes by varying the parameters of the potential. We extend our previous work on short soft-core ranges to the case of large soft-core ranges, by using an integral equation approach in the hypernetted-chain approximation. We show, using a modified van der Waals equation we recently introduced, that if there is a balance between the attractive and repulsive part of the potential this potential has two fluid-fluid critical points well separated in temperature and in density. This implies that for the repulsive (attractive) energy $U_{R}\left(U_{A}\right)$ and the repulsive (attractive) range $w_{R}\left(w_{A}\right)$ the relation $U_{R} / U_{A} \propto w_{R} / w_{A}$ holds for short soft-core ranges, while $U_{R} / U_{A} \propto 3 w_{R} / w_{A}$ holds for large soft-core ranges.
\end{abstract}

DOI: 10.1103/PhysRevE.71.061504 PACS number(s): 61.20.Gy, 61.20.Ne, 61.25.Mv, 64.60.Kw

\section{INTRODUCTION}

The phase diagram of a typical monatomic substance is comprised of solid and fluid phases, with the fluid phase separating below the critical point into gas and liquid phases. The prototype of such substances are simple (i.e., argonlike) fluids. Interparticle interactions in these systems can be appropriately described by the well-known Lennard-Jones potential. Other simple models-such as those described by the hard-sphere square-well potential or by the hard-sphereYukawa potential—exhibit similar phase diagrams [1]. All these potentials consist of a short-range harshly repulsive core plus a longer-ranged attraction. New insights into the relationship between phase diagrams and interparticle interaction emerged recently from the finding that when the range of the attractive component is sufficiently small, the liquid phase and the gas-liquid critical point become metastable with respect to crystallization [2-9]. Shouldered potentialspotentials with a hard core and a finite repulsive shoulderexhibit more exotic phase diagrams [10]. Simulations and theories showed that such potentials may give rise to nontrivial phase behaviors, such as isostructural solid-solid transitions and liquid-liquid transitions [10-22]. The key to this complex phase behavior resides in the peculiar penetrability of the repulsive core, a feature that gives rise to a densitydependent effective interaction.

The possible existence of a liquid-liquid phase transition for single-component systems with a standard gas-liquid critical point has received considerable attention in recent years. Direct evidence of this phenomenon has been observed experimentally in liquid phosphorus $[23,24]$ and triphenyl phosphite [25]. Experimental data consistent with a liquid-liquid phase transition have also been presented for other single-component systems such as water [26-28], silica [29,30], carbon [31], aluminate liquids [32], selenium [33], and cobalt [34], among others [35]. A liquid-liquid critical point has also been predicted by simulations for specific models of supercooled water [36-38], carbon [39], phosphorus [40], supercooled silica $[29,41,42]$, and hydrogen [43].
We have recently shown through molecular dynamics (MD) simulations $[15,19]$ that a system of particles interacting through an isotropic potential with an attractive well and a repulsive component consisting of a hard core plus a finite shoulder may possess a high-density liquid phase and a lowdensity liquid phase. Potentials with such characteristics were used to model interactions in a variety of systems including liquid metals, metallic mixtures, electrolytes and colloids, as well as anomalous liquids, like water and silica [44-53].

In spite of the simplicity of the model, the physical mechanism that causes the liquid-liquid transition for a potential with a hard core plus a repulsive shoulder and an attractive well is not easy to assess since it arises from an interplay of the different components of the pair interaction. To disentangle the role of each component it is necessary to investigate the dependence of the phase diagram on the potential parameters. This task was undertaken in Ref. [20], where the results of MD calculations performed for several sets of parameters were presented. The resulting behavior of the critical points was interpreted through a modified van der Waals equation (MVDWE) [20], a mean field approach assuming that the effect of the repulsive shoulder at different densities $\rho$ and temperatures $T$ can be taken into account by an effective excluded volume depending on both $\rho$ and $T$. However, the analysis was limited to cases where both the soft-core range and the attractive range are smaller than the hard-core range $a$ and the total interaction range does not exceed 2.6a. Nevertheless, there are cases such as biological solutions and colloids where the soft-core range could be as large as the hard-core or even larger [55]. For this reason, and to gain a better understanding of the role played by each component of a soft-core attractive potentials, we here explore how the phase diagram changes when the soft-core range exceeds the hard-core diameter.

We use an approach based on integral equations, which can be considered in many respects as an intermediate one between simulations and MVDWE. In fact, solving an integral equation is far less time consuming than a simulation, 
but is by no means as accurate. On the other hand, a microscopic theory is not based on the assumption typical of mean field approaches, such as the MVDWE, that particles experience a uniform attractive potential. Hence it is intrinsically more accurate.

In particular, we use the hypernetted-chain (HNC) integral equation for the radial distribution function [54] to estimate the position of the critical points. We perform an extensive investigation in the space of the potential parameters, considering an extremely ample number of combinations. This makes it possible to frame previous results into a wider perspective and allows a better understanding of the physical mechanism leading to a liquid-liquid transition in onecomponent fluids.

Our results show that the high-density critical point can be found only when there is a balance between the attractive part and the repulsive part of the potential. In Ref. [20] this balance was expressed through the mean field strength of attraction, a parameter related to the second virial coefficient, proportional to the attractive range $w_{A} / a$ and inversely proportional to the repulsive energy $U_{R}$, for fixed attractive energy $U_{A}$. Here we find an approximate relation between $U_{R} / U_{A}$ and $w_{A} / w_{R}$ (where $w_{R}$ is the repulsive range) that quantifies the ideal balance between the repulsive and the attractive components of the potential more effectively. Our results show that the liquid-liquid phase transition can be found in systems with small repulsion if the attraction is small as well, with $U_{R} / U_{A} \propto w_{A} / w_{R}$, and in systems with wide repulsion, with $U_{R} / U_{A} \propto 3 w_{A} / w_{R}$. Typical systems with these characteristics are colloids, where the effective repulsion and attraction can be regulated [57].

\section{THE ATTRACTIVE SOFT-CORE POTENTIAL}

A soft-core potential with an attractive interaction at large distances was first proposed, and studied through an exact analysis in 1D, by Hemmer and Stell [44] to understand the possibility of the solid-solid critical point in materials such as $\mathrm{Ce}$ and Cs. Other soft-core potentials with an attractive well were proposed and studied with approximate methods or with numerical simulations to rationalize the properties of liquid metals, alloys, electrolytes, colloids, and water anomalies [15-22,45-53].

The peculiarity of such potentials is the presence of two repulsive length scales. This feature is typical of systems with core-corona architecture such as, e.g., star polymers. However, isotropic soft-core potentials have also been proposed as effective potentials resulting from an average over the angular degrees of freedom for systems where the distance of the minimum approach between particles depends on their relative orientation. Thus, in some respect, they have been considered $[50,52,53]$ as simplified models of complex anisotropic interactions, such as those resulting from the hydrogen bonding between water molecules.

The model potential considered in this paper is similar to that investigated in Refs. [15,16,19-21]. It is an isotropic pair potential with two characteristic short-range repulsive distances: one associated with the hard-core exclusion between two particles and the second with a weak repulsion

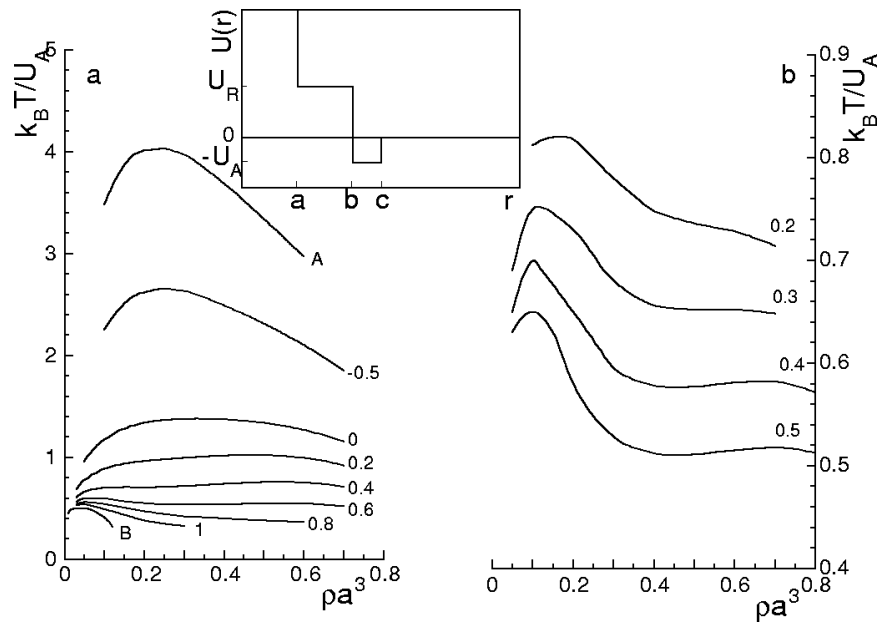

FIG. 1. Inset: General shape of the attractive soft-core potential used in this work, with hard-core distance $a$, soft-core distance $b$, interaction range $c$, attractive energy $U_{A}$, and repulsive energy $U_{R}$. Panels: Instability line of the HNC equation for the potential in the inset with $w_{A} / a=0.2$ : (a) $w_{R} / a=1$ and (from top to bottom) $U_{R} / U_{A}=-1$ (potential $A$ ), $-0.5,0,0.2,0.4,0.6,0.8,1, \infty$ (potential $B$ ); (b) $w_{R} / a=0.8$, and $U_{R} / U_{A}=0.2,0.3,0.4,0.5$.

("soft core"), which can be overcome at large pressure. More precisely, our pair potential $U(r)$ [Fig. 1(a), inset] consists of a hard core of radius $a$, a repulsive square shoulder of height $U_{R}$ extending from $r=a$ to $r=b$, and an attractive component having the form of a square well of energy $-U_{A}<0$ extending from $r=b$ to $r=c$ (here $r$ is the interparticle distance). Choosing $a$ and $U_{A}$ as length and energy units, respectively, this potential depends on three free parameters: the width of the soft core $w_{R} / a \equiv(b-a) / a$, the width of the attractive well $w_{A} / a \equiv(c-b) / a$, and the soft-core energy $U_{R} / U_{A}$.

Our aim is to understand how the position of the critical points in the thermodynamic plane changes upon varying parameter values. In Ref. [20] we investigated, using MD simulations, a number of cases with $w_{R}<a$ and $w_{A}<a$, and presented a mean field approach with an MVDWE to interpret the results. However, simulations (both MD or Monte Carlo) of potentials with $w_{R} \geqslant a$ require very large computation times, so we study this case with integral equations (in the HNC approximation) which represents a compromise between accuracy and economy.

\section{THE HYPERNETTED CHAIN INTEGRAL EQUATION APPROACH}

The spatial distribution of a system of particles may be conveniently described by the radial distribution function $g(r)$ [56], a quantity directly measurable by scattering experiments and related to the thermodynamic properties of the fluid. One of the theoretical approaches most used to calculate this function is represented by integral equations. These are based on the Ornstein-Zernike $(\mathrm{OZ})$ relation between the total pair correlation function $h(r) \equiv g(r)-1$ and the direct correlation function $c(r)$, which describes the contribution coming from the direct interaction between two particles 
separated by distance $r$. Both $h(r)$ and $c(r)$ are unknown functions, so to solve the OZ relation one needs another relation between these two functions (closure), which is necessarily approximate. Depending on the closure, one gets different equations. Examples include the Percus-Yevick, the HNC equations, the mean spherical approximation (MSA), etc. [56]. Their specific performance depends on the kind of intermolecular interaction as well as on the thermodynamic conditions, with no clear overall superiority of one theory. Moreover, all theories are affected by a thermodynamical inconsistency that can be partially removed through suitable modifications of the equations (which, however, makes their solution considerably less rapid).

For each integral theory there is a region in the $\rho-T$ plane where no solution can be found, i.e., for any $\rho$, there is a $T$ below which it is not possible to solve the equation. This defines an instability line (IL) in the $\rho$ - $T$ plane. Usually, the IL cannot be identified with the spinodal line of the fluid since, except for the MSA, it is not characterized by a truly diverging compressibility [58]. In any case, due to the thermodynamical inconsistency of the theory, the maximum of the spinodal line does not coincide with the maximum of the binodal line, so is not possible to obtain an unequivocal estimate of the critical point.

In spite of the above limitations, knowledge of the IL may allow us to estimate the topology of the region of spinodal decomposition of the fluid. In particular, it was found that the $\mathrm{IL}$ of the HNC equation for the potential defined in Sec. II, with parameters $w_{R} / a=1, w_{A} / a=0.2$, and $U_{R} / U_{A}=0.5$, is qualitatively similar to the spinodal line calculated through MD calculations [19]. More precisely, the density and temperature of the low-density critical point estimated through the HNC equation are in satisfactory agreement with simulation results, while the density of the second-critical point is overestimated by the theory [19]. This is not surprising since the theory is an approximate one and becomes progressively less accurate as the density increases. However, the ability of the HNC equation to give account of the presence of two critical points is, within the well-known limitations of the theory, quite remarkable since the potential considered gives origin to a phase diagram that is definitely unusual for simple fluids. Thus, studying the modifications of the IL as the potential parameters are varied can yield approximate, yet useful, information on the phase behavior of the fluid. In our calculations, we obtain the solution of the system formed by the $\mathrm{OZ}$ relation plus the $\mathrm{HNC}$ closure through a numerical iterative procedure using a grid with $M=2048$ discrete points, $r_{m}=m \delta r$, with $m=1, \ldots, M$, and $\delta r / a=0.01$.

\section{INSTABILITY LINES FOR LARGE REPULSIVE RANGE}

To disentangle the role of each component of the interparticle interaction, we vary the parameters of the potential one at a time. First we keep the width $w_{R}$ of the repulsive shoulder and the width $w_{A}$ of the attractive well fixed, and study the behavior of the instability of the IL by letting the height $U_{R}$ of the repulsive shoulder vary. The considered values of $U_{R}$ range from $-U_{A}$ to $\infty$. When $U_{R} / U_{A}=-1$, the potential consists of a hard core of radius $a$ and a square well of width $c-a$ (henceforth called potential $A$ ) whereas, when $U_{R} \rightarrow \infty$, the potential has a hard core of radius $b$ and a square well of width $c-b$ (potential $B$ ). When the shoulder height increases, starting from $U_{R} / U_{A}=-1$, the potential gradually changes from potential A to potential B. In any intermediate configuration, the potential has a penetrable finite repulsive shoulder.

The IL is shown in Fig. 1(a) at fixed shoulder and well widths $\left(w_{R} / a=1, w_{A} / a=0.2\right)$ for several values of the shoulder height $U_{R}$. In the two limiting cases corresponding to potentials $\mathrm{A}$ and $\mathrm{B}$, the IL exhibits a single maximum corresponding to a phase diagram with a single liquid-gas critical point, a well-known behavior for a fluid of hard spheres with an attractive well. The position of the critical points in the $\rho, T$ plane is considerably different in the two cases. The critical point corresponding to potential $\mathrm{B}$ is at a lower temperature than that corresponding to potential $\mathrm{A}$, due to the weaker attraction, i.e., shorter attractive range $c-b$ of potential $\mathrm{B}$ with respect to the largest attractive range $c-a$ of potential A. Furthermore, the critical density for potential B is smaller than that for potential A and rescales as the hardcore volume $(a / b)^{3}$ of the two potentials. We observe that, unless $b / a \simeq 1$, this rescaling overshadows the shift of the critical point toward higher densities due to the decrease of the attraction range (e.g., see Appendix A in Ref. [20]).

As $U_{R}$ increases, starting from $U_{R} / U_{A}=-1$ (potential A), the IL moves toward lower temperatures as a consequence of the overall reduction of the interparticle potential's attractive component. At the same time, the IL undergoes a change which eventually yields a line with two maxima [see the enlarged view in Fig. 2(b)]. This peculiar topology of the IL becomes most evident for intermediate values of $U_{R}(0.4$ $\leqslant U_{R} / U_{A} \leqslant 0.6$ ). As $U_{R}$ increases further, the second maximum disappears and again the shape of the IL becomes more and more similar to the shape typical of the hard-core squarewell potential. Thus, when $w_{R}$ and $w_{A}$ are fixed, two maxima are observed in the IL only for a finite range $U_{R}^{\max } \leqslant U_{R}$ $\leqslant U_{R}^{\min }$

In this range of values, as $U_{R}$ increases, the density $\rho_{1}$ of the low-density maximum becomes smaller, while that of the other maximum $\rho_{2}$ slightly increases. The critical temperatures $T_{1}$ and $T_{2}$, respectively, corresponding to these two maxima, decrease- this behavior being more evident for the second maximum. These results agree with the behavior found with MD simulations for the two critical points (reported in Fig. 9g, 9h of Ref. [20]). Thus for increasing $U_{R}$, the two maxima move away from each other both in density and temperature.

In Fig. 1(b) we show the behavior of the IL for $w_{R} / a$ $=0.8$ and $w_{A} / a=0.2$. Comparing these results with those shown in Figs. 1(a) and 2(b), we observe that for fixed $w_{A}$ and $U_{R}$, as $w_{R}$ increases, $\rho_{1}$ and $T_{1}$ are almost constant and at variance with MD results, but $\rho_{2}$ decreases and $T_{2}$ increases in agreement with the MD simulations (see Figs. 9d, 9e of Ref. [20]).

We now consider a fixed shoulder width $\left(w_{R} / a=1\right)$ and several values of the well width $\left(w_{A} / a=0.1,0.2,0.3,0.4\right.$, $0.5,0.6)$. We calculate the IL for each of them, letting the height $U_{R}$ of the shoulder vary [Figs. 2(a)-2(f)]. The values 

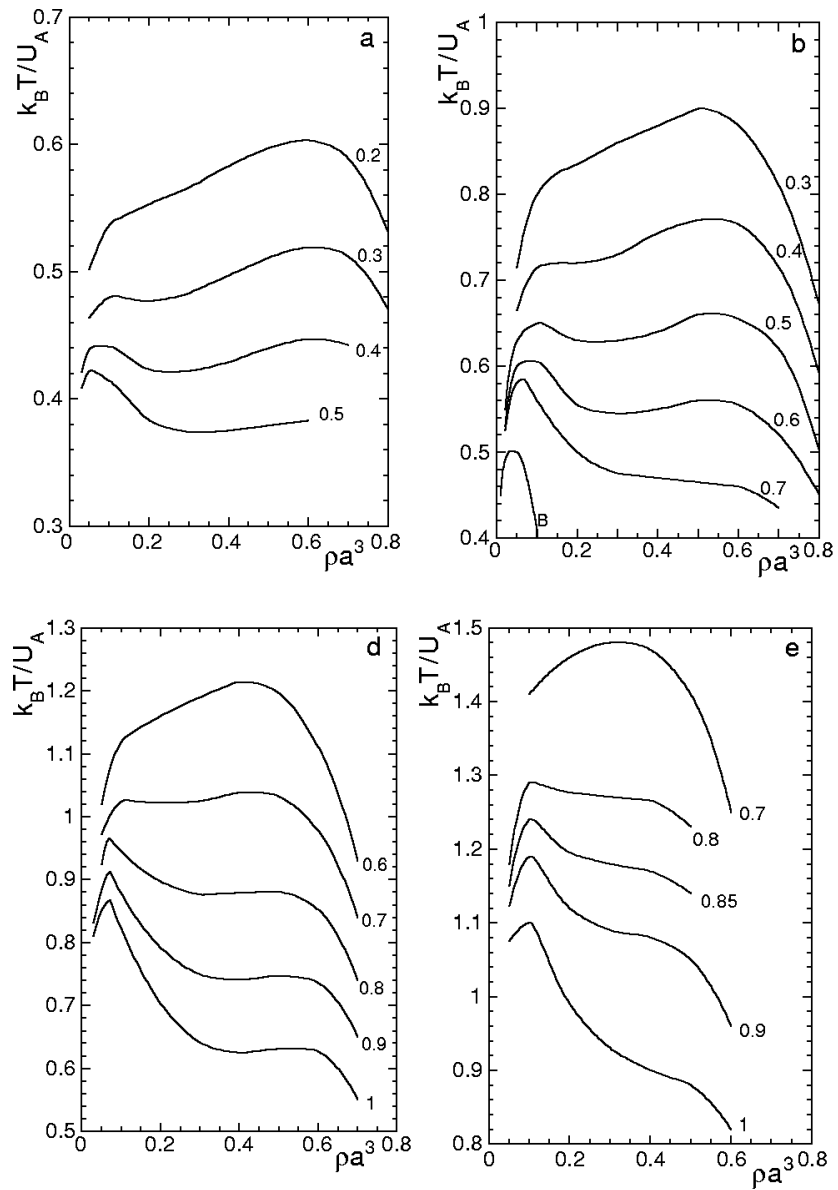

of the shoulder height $U_{R}$ for which two maxima are observed increase with $w_{A}$. By increasing $w_{A}$, the second maximum is less and less evident and for large values of $w_{A} / a$ [Figs. 2(e) and 2(f)] the second maximum is not observed for any $U_{R}$. For small values of $w_{A}$, the decrease of the attraction flattens the curve and the second maximum becomes difficult to observe [Fig. 2(a)].

For IL's with two maxima and the same $w_{R} / a=1$ and $U_{R}$ but different $w_{A}$, both maxima move toward higher temperatures for increasing $w_{A}$, due to the increased attraction. Moreover, by increasing $w_{A}, \rho_{2}$ becomes smaller while $\rho_{1}$ does not vary significantly. This behavior agrees with that predicted by MD simulations for the two critical points (shown in Figs. 9a, 9b of Ref. [20]).

We next consider a potential with a wider repulsive shoul$\operatorname{der}\left(w_{R} / a=1.5\right)$ and several values of the well width $\left(w_{A} / a\right.$ $=0.5,1.0,1.5,2.5)$. The behavior of the IL (Fig. 3) for varying $U_{R}$ is quite similar to that observed in the previous cases. For fixed $w_{R}$ and $w_{A}$, the IL shows only two maxima in a finite range of values of $U_{R}$; these values increase with $w_{A}$ and, for large values of $w_{A}$, the two-maxima topology is not observed regardless of the value of $U_{R}$ [Fig. 3(d)]. The range of values of $w_{A}$ in which we observe two maxima is larger with respect to the case $w_{R} / a=1$.

For one particular set of parameters $\left(w_{R} / a=1.5, w_{A} / a\right.$ $=0.5$ and $\left.U_{R} / U_{A}=0.8\right)$, it is possible to compare the results obtained using the HNC equation with the phase diagram calculated through a theoretical approach based on a thermo-

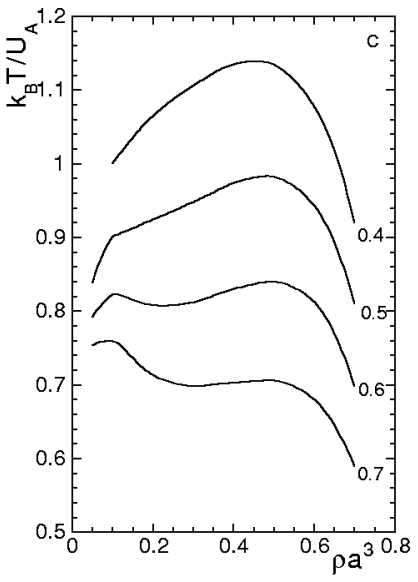

FIG. 2. Instability line of the HNC equation for $w_{R} / a=1$ and six values of the parameter $w_{A} / a$ : (a) $w_{A} / a=0.1$, and $U_{R} / U_{A}=0.2$, $0.3,0.4,0.5$; (b) $w_{A} / a=0.2$, and $U_{R} / U_{A}=0.3,0.4,0.5,0.6,0.7, \infty$ (potential B); (c) $w_{A} / a=0.3$, and $U_{R} / U_{A}=0.4, \quad 0.5, \quad 0.6,0.7$; (d) $w_{A} / a=0.4$, and $U_{R} / U_{A}=0.6,0.7$, $0.8,0.9,1$; (e) $w_{A} / a=0.5$, and $U_{R} / U_{A}=0.7,0.8,0.85,0.9,1$; (f) $w_{A} / a=0.6$, and $U_{R} / U_{A}=0.6,0.8$, $0.9,1,1.1, \infty$ (potential B). dynamically consistent integral equation [16]. Once again it appears evident that the main flaw of the HNC equation is to overestimate the critical density of the second critical point.

However, a direct comparison of HNC results with those obtained through MD simulations can be disappointing. For some of the parameter sets investigated in Fig. 9 of Ref. [20] the IL shows only one maximum, while for others the twomaxima topology is barely observable. As an example, we show the IL corresponding to the parameters $w_{R} / a=0.5$, $w_{A} / a=0.5$ with $1.0 \leqslant U_{R} / U_{A} \leqslant 1.7$ (Fig. 4). It was not possible to directly analyze the value $U_{R} / U_{A}=2$ (considered in Ref. [20]) since, in this case, the HNC cannot be solved at high densities before any considerable increase of the compressibility can be observed (in general, this occurs when the finite repulsion is considerably stronger than the attraction). The results obtained at slightly smaller values of $U_{R}$ show, however, a non-monotonic behavior of the IL, consistent with the presence of a liquid-liquid critical point.

\section{DISCUSSION}

The overall behavior of the IL's is synthesized in Fig. 5(a) which shows, for different values of the shoulder width $\left(w_{R} / a=0.6,0.8,1.0,1.5\right)$, the points in the $\left(w_{A}, U_{R}\right)$ plane where two maxima are found by using the HNC approximation. We observe that both ranges of $w_{A}$ and $U_{R}$ where two maxima are observed increase with $w_{R}$. 

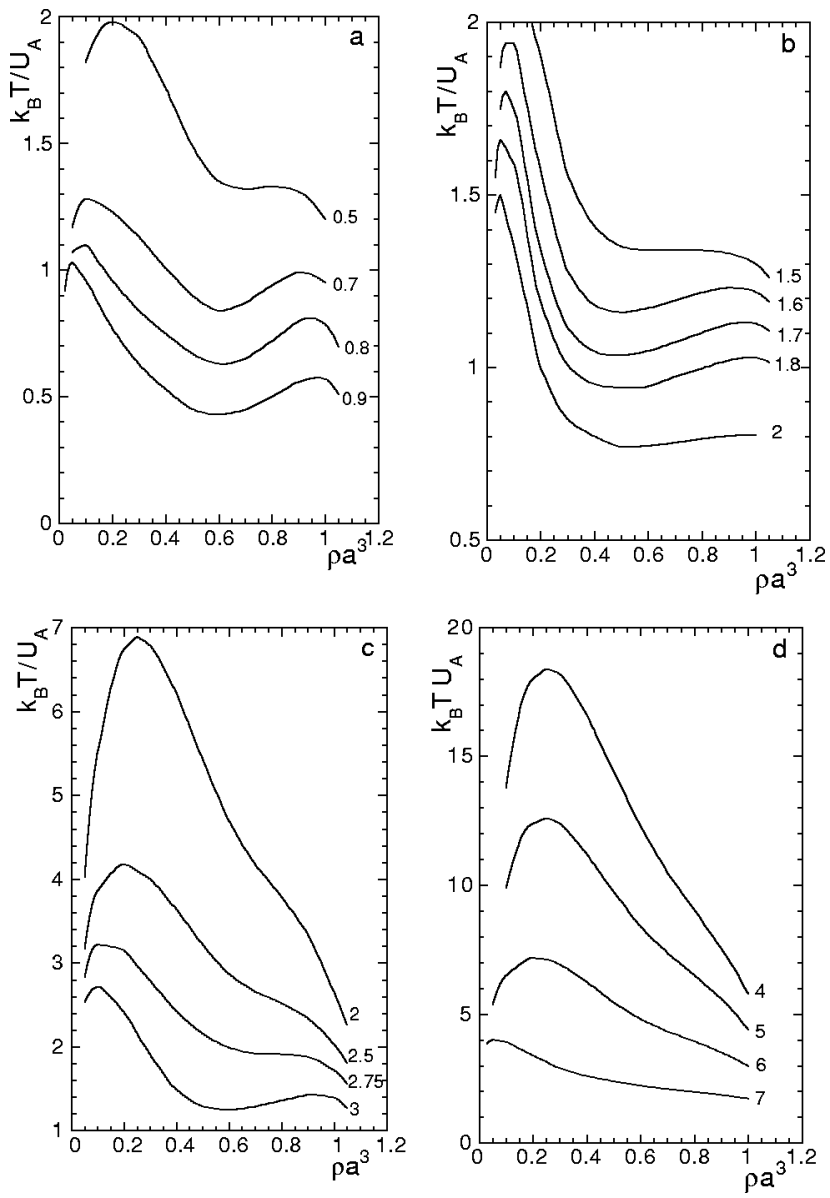

FIG. 3. Instability line of the HNC equation for $w_{R} / a=1.5$ and four values of the parameter $w_{A} / a$ : (a) $w_{A} / a=0.5$, and (from top to bottom) $U_{R} / U_{A}=0.5,0.7,0.8,0.9$; (b) $w_{A} / a=1$, and $U_{R} / U_{A}=1.5$, 1.6, 1.7, 1.8, 2; (c) $w_{A} / a=1.5$, and $U_{R} / U_{A}=2,2.5,2.75,3$; (d) $w_{A} / a=2.5$, and $U_{R} / U_{A}=4,5,6,7$.

The general behavior of $U_{R}$ as a function of $w_{A}$ at constant $w_{R}$ can be rationalized by using the modified van der Waals approach (MVDWE) presented in Ref. [20]. First we ap-
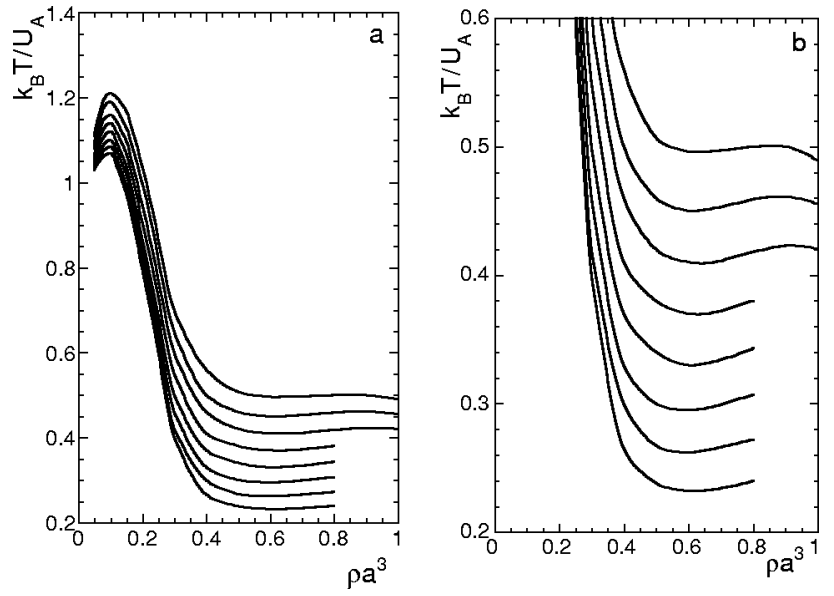

FIG. 4. Instability line of the HNC equation for (a) $w_{R} / a=0.5$, $w_{A} / a=0.5$, and (from top to bottom) $U_{R} / U_{A}=1,1.1,1.2,1.3,1.4$, 1.5, 1.6, 1.7; (b) an enlarged view at low $T$. proximate the interval of values of $U_{R}^{\min } \leqslant U_{R} \leqslant U_{R}^{\max }$ for each $w_{R}$ and $w_{A}$ in Fig. 5(a) with its middle point $U_{R}^{*}=\left(U_{R}^{\max }\right.$ $\left.+U_{R}^{\mathrm{min}}\right) / 2$ [Fig. 5(b)]. Next, we recall from Ref. [20] the relation between the potential's parameters and the strength of attraction $A$, a parameter related to the second virial coefficient $v_{2}$ and increasing with $w_{A} / a$ and decreasing with $U_{R} / U_{A}$. In particular, as $T \rightarrow \infty$ it is

$$
v_{2}=\frac{2 \pi}{3} a^{3}-\frac{A}{k_{B} T}+O\left(T^{-2}\right),
$$

with

$$
\begin{gathered}
A=U_{A} v_{A}-U_{R} v_{R}, \\
v_{A}=\frac{2 \pi}{3}\left[\left(a+w_{R}+w_{A}\right)^{3}-\left(a+w_{R}\right)^{3}\right],
\end{gathered}
$$

and

$$
v_{R}=\frac{2 \pi}{3}\left[\left(a+w_{R}\right)^{3}-a^{3}\right] .
$$

The relation $U_{R} / U_{A}=v_{A} / v_{R}-A /\left(U_{A} v_{R}\right)$ can be rewritten as

$$
\begin{aligned}
\frac{U_{R}}{U_{A}}= & \frac{V_{\mathrm{SC}}}{V_{\mathrm{SC}}-V_{\mathrm{HC}}}\left[-\frac{A}{U_{A} V_{\mathrm{SC}}}+3 \frac{R_{\mathrm{HC}}}{R_{\mathrm{SC}}} \frac{w_{A}}{a}+3 \frac{S_{\mathrm{HC}}}{S_{\mathrm{SC}}}\left(\frac{w_{A}}{a}\right)^{2}\right. \\
& \left.+\frac{V_{\mathrm{HC}}}{V_{\mathrm{SC}}}\left(\frac{w_{A}}{a}\right)^{3}\right],
\end{aligned}
$$

where

$$
\begin{aligned}
V_{\mathrm{HC}} & =\frac{2 \pi}{3} a^{3}, V_{\mathrm{SC}}=\frac{2 \pi}{3}\left(a+w_{R}\right)^{3}, \frac{S_{\mathrm{HC}}}{S_{\mathrm{SC}}}=\frac{a^{2}}{\left(a+w_{R}\right)^{2}}, \frac{R_{\mathrm{HC}}}{R_{\mathrm{SC}}} \\
& =\frac{a}{a+w_{R}}
\end{aligned}
$$

are the volumes, and the ratios of the surfaces and radii of the hard core (HC) and the soft core (SC), respectively, and all depend only on the parameter $w_{R} / a$. Hence, at a fixed value of $w_{R}$, the function $U_{R}\left(w_{A}\right)$ in Eq. (5) only has $A$ as an unknown parameter.

This MVDWE prediction can be verified by using the HNC results. In Fig. 5(b), Eq. (5) is used to fit the values of $U_{R}^{*}\left(w_{A}\right)$ resulting from $\mathrm{HNC}$ calculations for different values of $w_{R}$, with $A$ as the only fitting parameter. As expected from Eq. (5), when $w_{A} / a<1$, the leading order in $U_{R}^{*}\left(w_{A}\right)$ is linear, while when $w_{A} / a>1$ (corresponding to larger $w_{R}$ ), the nonlinear behavior is evident. Figure 5(b) also shows that, by increasing $w_{R}$, the coefficients of the third-degree polynomial in $w_{A}$ decrease as predicted by Eqs. (5) and (6).

Moreover, the fitting parameter $A$ in Fig. 5(b) shows a nonmonotonic behavior with $w_{R}$. This is consistent with the MVDWE prediction in Ref. [20] that $\partial A / \partial w_{R}$ may have different signs, depending on the other parameters. Therefore, Eqs. (5) and (6) give us a fair description of how the three parameters $U_{R}, w_{R}$, and $w_{A}$ are related to each other when the phase diagram has two critical points at positive pressure and finite temperature. However, Eqs. (5) and (6) do not help us understand why the phase diagram has an accessible liquid- 
liquid critical point only for limited ranges of $w_{A}$ and $U_{R}$, given a value of $w_{R}$.

To gain some insight into this point, we observe that if we plot Eq.(5) with $A=0$ and no fitting parameters [Fig. 5(c)], we get a rough approximation of the calculated $U^{*}$ that becomes fair for the largest $w_{R}$. This suggests that as a first approximation we can assume that $A=0$ at least for $w_{R} / a$ $>1$, which is consistent with the conclusion of Ref. [20] that, in order to have two accessible critical points in the fluid phase, the attractive and repulsive part of the potential must compensate, i.e., $U_{A} v_{A} \simeq U_{R} v_{R}$ or $A \simeq 0$.

Hence, from Eqs. (3) and (4) we get the approximation

$$
\frac{U_{R}}{U_{A}}=\frac{\left(a+w_{R}+w_{A}\right)^{3}-\left(a+w_{R}\right)^{3}}{\left(a+w_{R}\right)^{3}-a^{3}} .
$$

First we observe that to get an accessible liquid-liquid critical point, $U_{R} / U_{A} \sim O(1)$ is the relevant case. Indeed, the case with $U_{R} / U_{A} \gg 1$ at high-enough $T$ and small-enough $P$ corresponds to an effective attractive potential with no repulsive shoulder and a hard core at a distance of $a+w_{R}$ with no liquid-liquid phase transition, or with a liquid-liquid phase transition at vanishing $T$ and very high $P$ (see MD results in Figs. 9h, 9i in Ref. [20]). On the other hand, for $U_{R} / U_{A}$ $\simeq 0$, Eq. (7) gives $w_{A} \simeq 0$, leading to a simple hard-core potential with no attractive well. Hence we consider the case with $U_{R} / U_{A} \sim O(1)$.

Next, we observe that for increasing $w_{R}\left(w_{R} \rightarrow \infty\right)$, Eq. (7) becomes

$$
\frac{U_{R}}{U_{A}}=\left(1+\frac{w_{A}}{a+w_{R}}\right)^{3}-1,
$$

from which the condition $U_{R} / U_{A} \simeq 3 w_{A} / w_{R}$ follows for large $w_{R} / w_{A}$. This relation is reasonably satisfied by HNC data for $w_{R} / a=1\left(w_{A} / a \simeq 0.4\right.$ for $\left.U_{R} / U_{A} \simeq 0.9\right)$ and is better approximated for $w_{R} / a=1.5\left(w_{A} / a \simeq 0.5\right.$ for $\left.U_{R} / U_{A} \simeq 0.9\right)$. We can deduce from these considerations that to get a phase diagram with two accessible critical points in the fluid region, the three parameters of the potential should be related by the approximate relation Eq. (8) for $w_{R} / a \gg 1$, which reduces to $w_{A} \simeq w_{R} / 3$ for $U_{R} / U_{A} \simeq 1$.

\section{CONCLUSIONS}

The purpose of the present investigation has been to understand the role that the different components of the interparticle interaction play in the physical mechanism underlying the liquid-liquid phase transition in one-component systems. Thus we have investigated the phase diagram associated with an isotropic pair potential with an attractive well and a repulsive shoulder, by analyzing for which combinations of the potential parameters the phase diagram shows two critical points in the fluid phase. In a first paper [20] we used MD simulations and found limited ranges of the parameters so that the liquid-liquid phase transition was accessible. We also presented a general description based on the MVDWE approach, which rationalized our MD results.

We complete this analysis here by adopting a different approach with well-known limitations, but extremely fast in

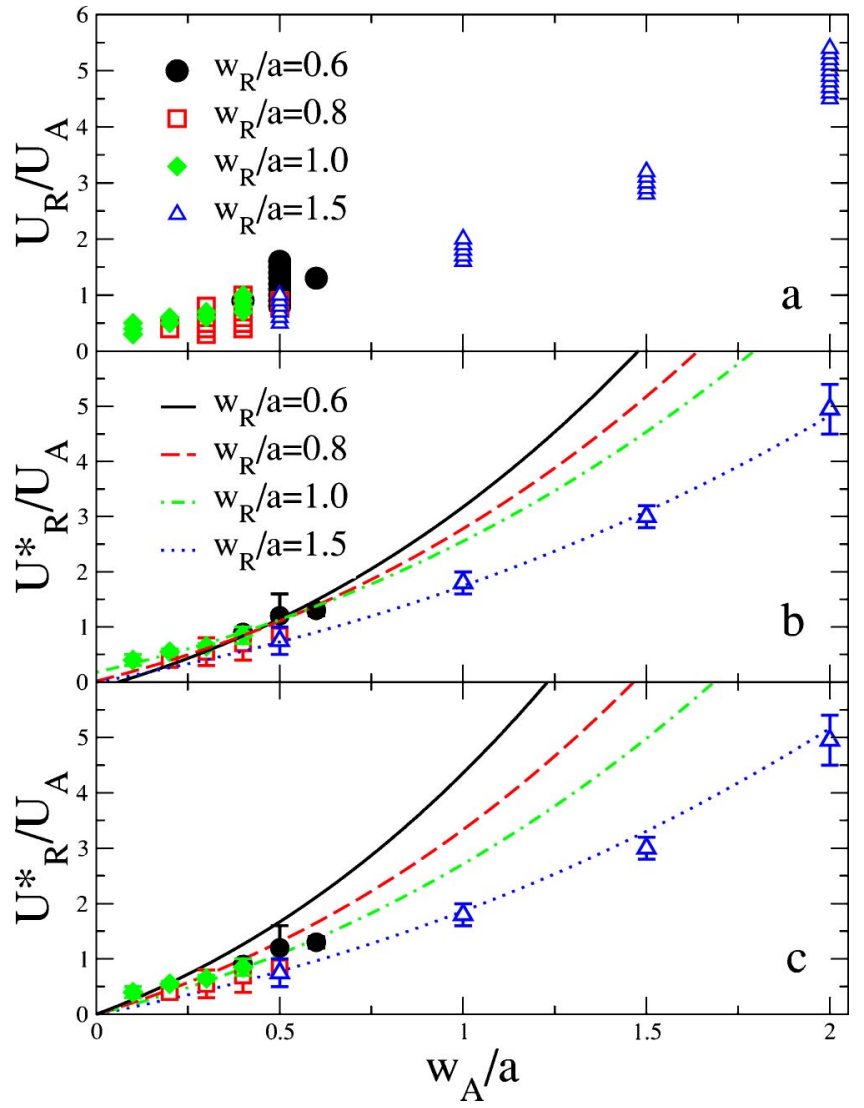

FIG. 5. (a) Symbols mark the combinations of the potential's parameters where the instability line, calculated by the HNC approach, shows two maxima, suggesting the presence of two fluidfluid critical points. Sets with $w_{R} / a=0.6$ (circles) were investigated for $0 \leqslant U_{R} / U_{A} \leqslant 2$ and $0 \leqslant w_{A} / a \leqslant 1$; sets with $w_{R} / a=0.8$ (squares) and with $w_{R} / a=1.0$ (diamonds) for $0 \leqslant U_{R} / U_{A} \leqslant 1.2$ and 0 $\leqslant w_{A} / a \leqslant 0.6$; sets with $w_{R} / a=1.5$ (triangles) for $0 \leqslant U_{R} / U_{A} \leqslant 6$ and $0 \leqslant w_{A} / a \leqslant 3$. Parameters outside these regions have not been investigated. (b) Middle points $U_{R}^{*}$ of intervals of $U_{R}$ in panel (a). Symbols are as in panel (a). Error bars represent the intervals in panel (a). Lines are one-parameter fits with Eq. (5): for set 2 (squares) the fitting parameter is $A /\left(U_{A} V_{\mathrm{SC}}\right)=0.31$ (dashed line); for set 3 (diamonds) the fitting parameter is $A /\left(U_{A} V_{\mathrm{SC}}\right)=-0.84$ (dot-dashed line); for set 4 (triangles) the fitting parameter is $A /\left(U_{A} V_{\mathrm{SC}}\right)=2.23$ (dotted line). Since we only have three points for set 1 , to avoid a fit with a large indeterminacy on the parameters we arbitrarily chose $A /\left(U_{A} V_{\mathrm{SC}}\right)=1$ (solid line) to show that the data are consistent with Eq. (5). (c) Lines are Eq.(5) evaluated with $A=0$ for each set. Symbols and lines are as in panel (b).

terms of computational time, consisting of integral equations in the HNC approximation. It is important to stress that the drawbacks of the HNC equation are not critical for our purposes. Indeed, we find that the theory, though at best only in qualitative agreement with MD simulations, correctly reproduces the trend according to which the simulated critical points move in the $\rho, T$ plane as the potential parameters are changed [20]. On this basis, we use the theoretical results to estimate the phase behavior of our system over a portion of the parameter space much wider than that explored by numerical simulations. 
Our findings, both with the MD and HNC approaches, show that only a limited number of combinations of potential parameters can be associated with a phase diagram with two accessible critical points in the fluid phase. A general conclusion is that the repulsive component of the potential must equilibrate the attractive component, i.e., that the strength of attraction, related to the second virial coefficient, is $A \simeq 0$, leading to Eq. (7). This equation gives us the intuitive understanding that the repulsive and attractive components of the interaction potential compensate when the attractive volume, weighted by the attractive energy, is equal to the repulsive volume, weighted by the repulsive energy.

For $w_{R} \gg a$ the MVDWE predictions for $A=0$ compare well with the HNC results [Fig. 5(c)], and for large $w_{R} / w_{A}$ Eq. (7) reduces to the simple Eq. (8), whose leading order is $3 w_{A} / w_{R}$. For $w_{R} \ll a$ it is difficult to extract a clear relation among the potential's parameters. However, we note that Eq. (5) shows a leading linear relation between $U_{R} / U_{A}$ and $w_{A} / w_{R}$ for $w_{R} \ll a$ and $w_{A} \ll a$, suggesting that the liquidliquid phase transition could also be found in systems with short repulsive range, if the attractive range is short as well.

Finally, for small attractive range $w_{A} / a$ the two fluid-fluid phase transitions generated by this potential are metastable with respect to the crystal $[15,19]$, consistent with what is expected on the basis of results for other short-range attractive potentials (see, e.g., Ref. [1]). Since for all these shortrange attractive potentials [59], including the present [60], the second virial coefficient is slightly negative [61] around the fluid-fluid critical point, from Eq. (1) this would imply $A>0$. We therefore expect that in general, for both large and short repulsive (and attractive) ranges, the condition $A /\left(U_{A} V_{\mathrm{SC}}\right) \geq 0$ would be verified for potentials with a phase diagram with two critical points. This condition could be generalized to

$$
1 \gtrsim 1 /\left(U_{A} V_{\mathrm{SC}}\right) \int_{0}^{\infty} U(r) d \vec{r} \gtrsim-2,
$$

for a continuous isotropic attractive potential $U(r)$ with a soft-core repulsion, where $a$ is an (effective) hard-core distance and $V_{\mathrm{SC}}$ is the soft-core volume defined in (6), both possibly $T$ dependent [62].

\section{ACKNOWLEDGMENTS}

We thank P. G. Debenedetti and F. Sciortino for fruitful discussions. G.F. acknowledges financial support from the Spanish Ministerio de Educación y Ciecia (Programa Ramón y Cajal and Grant No. FIS2004-03454) and the allocation of computer resources from INFM Progetto Calcolo Parallelo. We thank the NSF for financial support, through the computational chemistry program and the collaborative research in chemistry program.
[1] M. G. Noro and D. Frenkel, J. Chem. Phys. 113, 2941 (2000).

[2] A. P. Gast, C. K. Hall C. K. Elliot, and W. B. Russel, J. Colloid Interface Sci. 96, 251 (1983).

[3] H. N. W. Lekkerkerker, W. C. Poon, P. N. Pusey, A. Stroobants, and P. B. Warren, Europhys. Lett. 20, 559 (1992).

[4] C. F. Tejero, A. Daanoun, H. N. W. Lekkerkerker, and M. Baus, Phys. Rev. Lett. 73, 752 (1994).

[5] P. Bolhuis and D. Frenkel, Phys. Rev. Lett. 72, 2211 (1994).

[6] P. Bolhuis, M. Hagen, and D. Frenkel, Phys. Rev. E 50, 4880 (1994).

[7] P. Rein ten Wolde and D. Frenkel, Science 277, 1975 (1997).

[8] D. L. Pagan, M. E. Gracheva, and J. D. Gunton, J. Chem. Phys. 120, 8292 (2004); D. L. Pagan and J. D. Gunton, condmat/0412177.

[9] C. K. Hall and G. Stell, Phys. Rev. A 7, 1679 (1973).

[10] J. M. Kincaid, G. Stell, and C. K. Hall, J. Chem. Phys. 65, 2161 (1976); J. M. Kincaid, G. Stell, and E. Goldmark, ibid. 65, 2172 (1976); J. M. Kincaid and G. Stell, ibid. 67, 420 (1977).

[11] D. A. Young and B. J. Alder, Phys. Rev. Lett. 38, 1213 (1977); J. Chem. Phys. 70, 473 (1979).

[12] P. Bolhuis and D. Frenkel, J. Phys.: Condens. Matter 9, 381 (1997).

[13] E. Velasco, L. Mederos, G. Navascués, P. C. Hemmer, and G. Stell, Phys. Rev. Lett. 85, 122 (2000).

[14] P. C. Hemmer, E. Velasco, L. Mederos, G. Navascués, and G. Stell, J. Chem. Phys. 114, 2268 (2001).

[15] G. Franzese, G. Malescio, A. Skibinsky, S. V. Bulderev, and
H. E. Stanley, Nature (London) 409, 692 (2001).

[16] G. Malescio and G. Pellicane, Phys. Rev. E 63, 020501(R) (2001)

[17] S. Buldyrev, G. Franzese, N. Giovambattista, G. Malescio, M. R. Sadr-Lahijany, A. Scala, A. Skibinsky, and H. E. Stanley, Physica A 304,23 (2002).

[18] G. Malescio, G. Franzese, G. Pellicane, A. Skibinsky, S. V. Buldyrev, and H. E. Stanley, J. Phys.: Condens. Matter 14, 2193 (2002).

[19] G. Franzese, G. Malescio, A. Skibinsky, S. V. Buldyrev, and H. E. Stanley, Phys. Rev. E 66, 051206 (2002).

[20] A. Skibinsky, S. V. Buldyrev, G. Franzese, G. Malescio, and H. E. Stanley, Phys. Rev. E 69, 061206 (2004).

[21] B. Pellicane, G. Pellicane, and G. Malescio, J. Chem. Phys. 120, 8671 (2004).

[22] A. Lopes Balladares and M. C. Barbosa, J. Phys.: Condens. Matter 16, 8811 (2004); A. B. de Oliveira and M. C. Barbosa, ibid. 17, 399 (2005); V. B. Henriques and M. C. Barbosa, cond-mat/0407763.

[23] Y. Katayama, T. Mizutani, W. Utsumi, O. Shimomura, M. Yamakata, and K. Funakoshi, Nature (London) 403, 170 (2000); Y. Katayama, Y. Inamura, T. Mizutani, M. Yamakata, W. Utsumi, and O. Shimomura, Science 306, 848 (2004).

[24] G. Monaco, S. Falconi, W. A. Crichton, and M. Mezouar, Phys. Rev. Lett. 90, 255701 (2003).

[25] H. Tanaka, R. Kurita, and H. Mataki, Phys. Rev. Lett. 92, 025701 (2004); R. Kurita and H. Tanaka, Science 306, 845 (2004). 
[26] O. Mishima and H. E. Stanley, Nature (London) 396, 329 (1998); O. Mishima, Phys. Rev. Lett. 85, 334 (2000).

[27] M. C. Bellissent-Funel, Nuovo Cimento Soc. Ital. Fis., D 20D, 2107 (1998).

[28] A. K. Soper and M. A. Ricci, Phys. Rev. Lett. 84, 2881 (2000).

[29] C. A. Angell, S. Borick, and M. Grabow, J. Non-Cryst. Solids 207, 463 (1996); P. H. Poole, M. Hemmati, and C. A. Angell, Phys. Rev. Lett. 79, 2281 (1997).

[30] D. J. Lacks, Phys. Rev. Lett. 84, 4629 (2000).

[31] M. van Thiel and F. H. Ree, Phys. Rev. B 48, 3591 (1993).

[32] S. Aasland and P. F. McMillan, Nature (London) 369, 633 (1994); M. C. Wilding and P. F. McMillan, J. Non-Cryst. Solids 293, 357 (2001).

[33] V. V. Brazhkin, E. L. Gromnitskaya, O. V. Stalgorova, and A. G. Lyapin, Rev. High Pressure Sci. Technol. 7, 1129 (1998).

[34] M. G. Vasin and V. I. Ladýanov, Phys. Rev. E 68, 051202 (2003).

[35] P. F. McMillan, J. Mater. Chem. 14, 1506 (2004).

[36] P. H. Poole, F. Sciortino, U. Essmann, and H. E. Stanley, Nature (London) 360, 324 (1992).

[37] G. Franzese, M. I. Marques, and H. E. Stanley, Phys. Rev. E 67, 011103 (2003); G. Franzese and H. E. Stanley, Physica A 314, 508 (2002); G. Franzese and H. E. Stanley, J. Phys.: Condens. Matter 14, 2201 (2002).

[38] F. Sciortino, E. La Nave, and P. Tartaglia, Phys. Rev. Lett. 91, 155701 (2003).

[39] J. N. Glosli and F. H. Ree, Phys. Rev. Lett. 82, 4659 (1999).

[40] T. Morishita, Phys. Rev. Lett. 87, 105701 (2001).

[41] I. Saika-Voivod, F. Sciortino, and P. H. Poole, Phys. Rev. E 63, 011202-1 (2001).

[42] S. Sastry and C. A. Angell, Nature Mater. 2, 739 (2003).

[43] S. Scandolo, Proc. Natl. Acad. Sci. U.S.A. 100, 3051 (2003).

[44] P. C. Hemmer and G. Stell, Phys. Rev. Lett. 24, 1284 (1970); G. Stell and P. C. Hemmer, J. Chem. Phys. 56, 4274 (1972).

[45] P. G. Debenedetti, Metastable Liquids: Concepts and Principles (Princeton University Press, Princeton, NJ, 1998); Hydration Processes in Biology. Theoretical and Experimental Approaches, Vol. 305 of NATO Advanced Studies Institute, Series A: Life Sciences, edited by M. C. Bellissent-Funel (IOS Press, Amsterdam, 1998).

[46] M. Silbert and W. H. Young, Phys. Lett. 58A, 469 (1976); D. Levesque and J. J. Weis, ibid. 60A, 473 (1977); J. M. Kincaid and G. Stell, ibid. 65A, 131 (1978).

[47] W. M. Shyu, J. H. Wehling, and M. R. Cordes, Phys. Rev. B 4, 1802 (1971); M. Appapillai and V. Heine, Cavendish Laboratory Technical Report No. 5, 1972 (unpublished); K. K. Mon, N. W. Ashcroft, and G. V. Chester, Phys. Rev. B 22, 5014
(1980).

[48] J. M. Lawrence, M. C. Croft, and R. D. Parks, Phys. Rev. Lett. 35, 289 (1975).

[49] P. G. Debenedetti, V. S. Raghavan, and S. S. Borick, J. Phys. Chem. 95, 4540 (1991).

[50] F. H. Stillinger and T. Head-Gordon, Phys. Rev. E 47, 2484 (1993).

[51] S. S. Borick, P. G. Debenedetti, and S. Sastry, J. Phys. Chem. 99, 3781 (1995); T. M. Truskett, P. G. Debenedetti, S. Sastry, and S. Torquato, J. Chem. Phys. 111, 2647 (1999).

[52] M. R. Sadr-Lahijany, A. Scala, S. V. Buldyrev, and H. E. Stanley, Phys. Rev. Lett. 81, 4895 (1998); Phys. Rev. E 60, 6714 (1999); A. Scala, M. R. Sadr-Lahijany, N. Giovambattista, S. V. Buldyrev, and H. E. Stanley, ibid. 63, 041202 (2001); J. Stat. Phys. 100,97 (2000).

[53] E. A. Jagla, Phys. Rev. E 58, 1478 (1998); J. Chem. Phys. 111, 8980 (1999); Phys. Rev. E 63, 061501 (2001).

[54] J. M. J. Van Leeuwen, J. Groenveld, and J. De Boer, Physica (Amsterdam) 25, 792 (1959); G. S. Rushbrooke, ibid. 28, 259 (1960); E. Meeron, J. Math. Phys. 1, 192 (1960); T. Morita, Prog. Theor. Phys. 23, 829 (1960); L. Verlet, Nuovo Cimento 18,77 (1960).

[55] See, for example, F. Lo Verso, M. Tau, and L. Reatto, J. Phys.: Condens. Matter 15, 1505 (2003); C. N. Likos, N. Hoffmann, H. Lowen, and A. A. Louis, J. Phys.: Condens. Matter 14, 7681 (2002).M. Quesada-Pérez, A. Moncho-Jordá, F. Martínez-López, and R. Hidalgo-Álvarez, J. Chem. Phys. 115, 10897 (2001).

[56] J. P. Hansen and I. R. McDonald, Theory of Simple Liquids (Academic Press, London, 1976).

[57] M. M. Baksh, M. Jaros, and J. T. Groves, Nature (London) 427, 139 (2004).

[58] S. M. Foiles and N. W. Ashcroft, Phys. Rev. A 24, 424 (1981); P. D. Poll and N. W. Ashcroft, Phys. Rev. A 32, 1722 (1985); 35, 866 (1987); 35, 5167 (1987); L. Belloni, J. Chem. Phys. 98, 8080 (1993); J. S. Hoye, E. Lomba, and G. Stell, Mol. Mater. 79, 523 (1993).

[59] D. Rosenbaum, P. C. Zamora, and C. F. Zukoski, Phys. Rev. Lett. 76, 150 (1996); D. Rosenbaum and C. F. Zukoski, J. Cryst. Growth 169, 752 (1996).

[60] For the potential considered in $[15,19]$ is $v_{2} / v_{2}^{\mathrm{HS}} \simeq-4.68$ for the high density critical point and $v_{2} / v_{2}^{\mathrm{HS}} \simeq-6.41$ for the low density critical point, where $v_{2}^{\mathrm{HS}}$ is the hard-sphere second virial coefficient.

[61] A. George and W. W. Wilson, Acta Crystallogr., Sect. D: Biol. Crystallogr. D50, 361 (1994).

[62] J. A. Barker and D. Henderson, Rev. Mod. Phys. 48, 587 (1976). 